\title{
Frequency and Determinants of Peripheral Neuropathy in Diabetic Children in Sohag, Egypt
}

\author{
Montaser M. Mohamed, Rasha A. Ali*, Mohammed N. Hamdoon \\ Faculty of Medicine, Sohag University, Sohag, Egypt \\ Email: *rashaali815@yahoo.com
}

How to cite this paper: Mohamed, M.M., Ali, R.A. and Hamdoon, M.N. (2019) Frequency and Determinants of Peripheral Neuropathy in Diabetic Children in Sohag, Egypt. Journal of Behavioral and Brain Science, 9, 184-194.

https://doi.org/10.4236/jbbs.2019.94016

Received: March 28, 2019

Accepted: April 20, 2019

Published: April 23, 2019

Copyright $\odot 2019$ by author(s) and Scientific Research Publishing Inc. This work is licensed under the Creative Commons Attribution International License (CC BY 4.0).

http://creativecommons.org/licenses/by/4.0/

\begin{abstract}
Background: Diabetic peripheral neuropathy (DPN) is a common complication among children with TIDM and is related to poor glycemic control. The aim of the study is to determine prevalence and determinants of diabetic neuropathy in diabetic children. Material\& Methods: This is a cross sectional study conducted on 50 children having Type I diabetes mellitus for more than year presented to Pediatric Neurology and Endocrinology Outpatient Clinics, Sohag University Hospital, Sohag, Egypt, during one year duration (2017). Children were subjected to detailed neurological examination and electrophysiologic studies in addition to recording of socio demographic and laboratory findings with special regard to level of HbAlc. Results: The mean age of the studied children was $10.5 \pm 0.3$ years ranging from 5 to 18 years. 22 cases $(44 \%)$ were younger than 10 years, 27 children (54\%) were females. DPN was diagnosed in $12 \%$ of the studied children. No significant relation was found as regard age, gender, family history, consanguinity and duration of the diseases. Significant association was found between the HbAlc level and development of DPN in the studied children. Conclusion: Based on the results of this study, $12 \%$ of the studied diabetic children had DPN of whom more than $16 \%$ had subclinical neuropathy indicating the need for annual screening of neuropathy using Nerve Conduction Study. Moreover strict glycaemic control is needed to prevent rapid development of diabetic among these children.
\end{abstract}

\section{Keywords}

Diabetes, Mellitus, Children, Peripheral Neuropathy, HbA1c

\section{Introduction}

Type 1 diabetes is a chronic metabolic disease characterized by absolute insulin deficiency, resulting from a $\mathrm{T}$ cell-mediated destruction of pancreas B cell. It is 
considered one of the most common endocrine diseases in childhood and constitutes $5 \%-10 \%$ of patients with diabetes [1]. The annual incidence of T1DM varied from 0.61 cases per 100,000 populations in China to 57.6 cases per 100,000 in Finland [2]. Middle East region, Saudi Arabia and Kuwait have the highest incidence rate at 31.4 and 22.3/100,000 per year [3]. In Egypt, the prevalence of type I diabetes in adolescents was $0.6 \%$ and there is growing epidemic of diabetes among children and adolescents [4].

Diabetic neuropathy is known as a set of varied clinical syndromes with sensory, motor, and/or autonomic nerve fiber dysfunction, although the most common type is symmetrical distal sensory polyneuropathy (DPN) [5]. Diabetic peripheral neuropathy (DPN) is a common complication of T1DM, and unlike adults, patient's polyneuropathy has no clinical manifestations in diabetic children in its early stages [6]. Clinical examination had less sensitivity and specificity than nerve conduction studies, which serves as a gold standard to detect subclinical neuropathy in these patients [7]. DPN is a major cause of morbidity and poor quality of life in patients with diabetes mellitus. It can increase the chances of other complications, namely amputation and foot ulcer [8].

Abnormalities of nerve conduction are common findings in children with diabetes. The prevalence of DPN in both adults and children ranges between $7 \%$ and $57 \%(8 \mathrm{~m})$, and the highest prevalence for DPN is found in children and adolescents with poor glycemic control and longer duration of diabetes, abnormal lipid profile, which are considered important risk factors for DPN [9] [10].

Patients with TIDM have subclinical neuropathy long before occurrence of evident neuropathy, Moreover once symptoms appear, there are few effective therapeutic strategies [11] [12]. This indicates importance of early identification of DPN, in its primary stages to prepare appropriate measures to prevent its development. However, few studies have evaluated the subclinical neuropathy in these patients, and also the prevalence of early stages of DPN among children and adolescents is not well-known.

The aim of this research was to evaluate the prevalence of DPN (both clinical and subclinical subtypes) and its possible risk-factors in children with insulin dependent DM.

\section{Material \& Method}

\subsection{Study Design}

This is a cross sectional study conducted to determine prevalence and determinants of DPN in diabetic children presented to Pediatric department in one year duration extending from October $1^{\text {st }} 2016$ to September $30^{\text {th }} 2017$.

\subsection{Setting}

Pediatric Neurology, Endocrinology Outpatient Clinics and Pediatric Department, Sohag University Hospital. 


\subsection{Study Population}

All patients from 5 to 18 years suffering from diabetes mellitus type 1 for more than one year presented to Neurology and Endocrine Outpatient Clinics during the period of the study were included in this study. Exclusion criteria included: diabetic patients with duration of diabetes less than 1 year, age younger than 5 years old or older than 18 years old, children with other chronic diseases, handicapped children, children with cancer, chronic renal impairment, or other neurological diseases and children who were on medications that can produce peripheral neuropathy, i.e. antineoplastic drugs. Accordingly 50 patients were included in the study.

\subsection{Data Collection Procedure}

Predesigned questionnaire was filled by the investigator from the child or his parents. The first section of the questionnaire included in addition to socio demographic data of the studied child (age, gender, parental consanguinity and residence), detailed clinical data with special reference to (age of onset, duration and complications of diabetes mellitus, the presenting symptoms focusing on symptoms of peripheral neuropathy as numbness, tingling, loss of sensation, ulceration and Family history of diabetes mellitus and diabetic neuropathy.

The second section included data of patients examination focusing on neurological system as detailed neurological examination. Vibration perception, temperature sensation, pinprick sensation, MMT and ankle reflex were also examined in the participants. A tuning fork $(128 \mathrm{~Hz})$, Semmes-Weinstein monofilament test, cold and warm water test, and a reflex hammer were used for the evaluations. Neuropathy was evaluated through electrodiagnostic studies. The reference for evaluation and comparison of the electro-diagnostic parameters of sensorimotor responses were based on the tables from the "Electrodiagnosis in diseases of the nerve and muscle" and "electro-diagnostic medicine".

The evaluation was done in a calm room with optimal lighting and temperature temperature was approximately 34 degrees Celsius, at the time of evaluation. The sensory nerves evaluated were the median, radial and ulnar nerves in the upper limbs, Sural and superficial peroneal nerves in the lower limbs. The methods used for the stimulation, recording, and analysis of the data were based on the standards of the books aforementioned. Nerve Conduction Velocity (NCV) and distal latency tests were performed in the following nerves: Common peroneal nerve, tibial nerve (motor), superficial peroneal nerve, and sural nerve (sensory) nerve in the lower extremities, as well as median (motor and sensory) nerves in the upper extremities, were observed in the tests. This section of the questionnaire included results of Hemoglobin A1C.

\subsection{Ethical Consideration}

Approval of Sohag Faculty of Medicine Research Ethics Committee was obtained. Written consent was obtained from the parents. 


\subsection{Statistical Analysis}

The statistical analysis was carried out using SPSS software for Windows (version 22.0, Chicago, IL, USA). Sample characteristics were summarized numbers and percentage for categorical variables. Chi-Square test was used for comparison between qualitative variables. The associations between DPN and both duration of diabetes and HbAlc level was done using Mann-Whitney test.

\section{Results}

The mean age of the studied children was $10.5 \pm 0.3$ years ranging from 5 to 18 years. Among the studied 50 children having TIDM 22 cases (44\%) were younger than 10 years, 27 children (54\%) were females and 25 children (50\%) were from urban areas. Family history of diabetes was found in 27 cases (54\%), while positive consanguinity was detected in 20 cases (40\%).

Among the studied children with type I diabetes $12 \%$ were diagnosed as having diabetic neuropathy as displayed in Figure 1. As regard the type of neuropathy, one case (2\%) subclinical neuropathy, two cases (4\%) had mild sensory axonal neuropathy, 2 cases (4\%) had mild sensory motor axonal neuropathy, and one case (2\%) had moderate sensory motor axonal neuropathy (Figure 2).

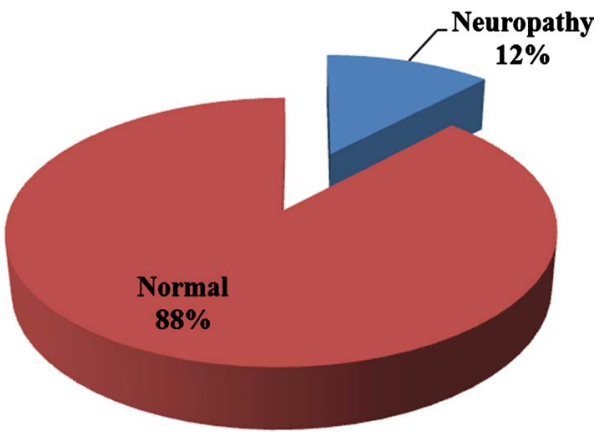

Figure 1. The percentage of neuropathy among the studied diabetic children.

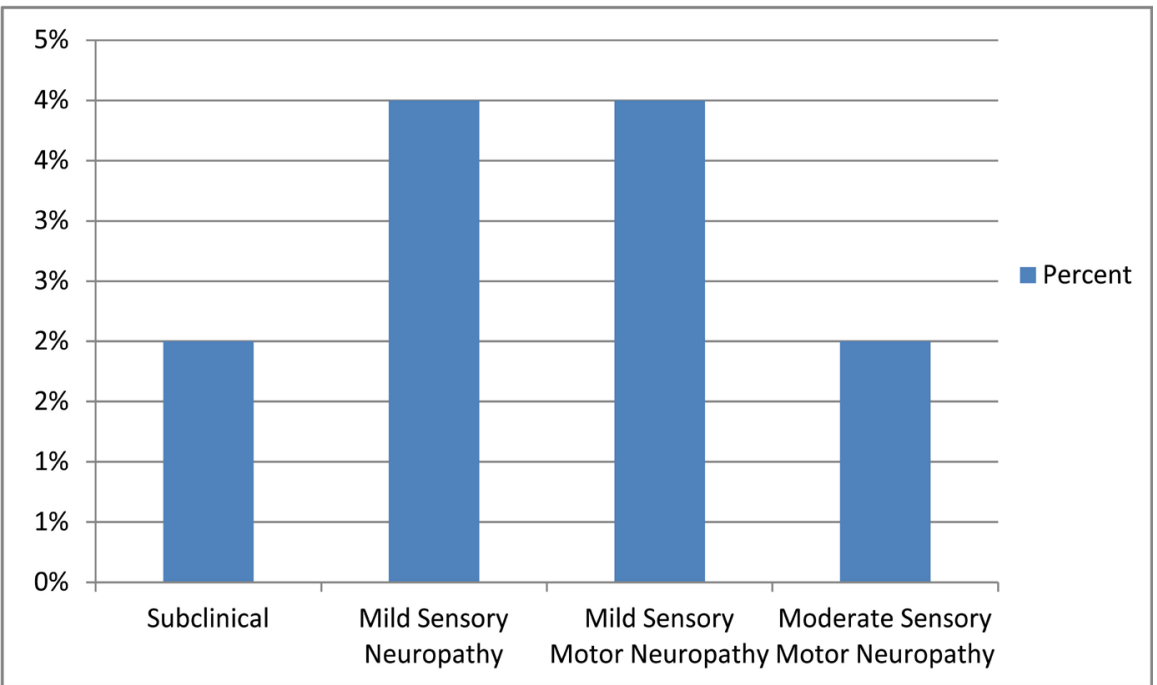

Figure 2. Distribution of the studied diabetic children according to type of neuropathy. 
As displayed in Table 1 no significant relation was found for age, gender, residence, consanguinity and family history and duration of diabetes and DPN. While highly significant association was found between DPN and Hemoglobin A1C.

Image 1: NCS show Rt. minimal sensory carpal tunnel syndrome (CTS) of a male diabetic patient 11 years old diagnosed 7 yeares ago was complaining of numbness and tingling in Rt. and Lt. hand, his serum creatinine was 0.8 , and $\mathrm{Hb}$

Table 1. Comparison between diabetic children with and without peripheral neuropathy as regard socio demographic characteristic, family history of diabetes, duration of diabetes and HbA1c level.

\begin{tabular}{cccc}
\hline Characteristics & Neuropathy $(\mathbf{N}=6)$ & Normal $(\mathbf{N}=44)$ & P-value \\
\hline Age & & $21(95.5 \%)$ & \\
$<10$ years & $1(4.5 \%)$ & $23(82.1 \%)$ & 0.211 \\
$\geq 10$ years & $5(17.9 \%)$ & $20(87 \%)$ & 1 \\
Gender & & $24(88.9 \%)$ & \\
Males & $3(13 \%)$ & & 1 \\
Females & $3(11.1 \%)$ & $22(88 \%)$ & 1 \\
Residence & & $22(88 \%)$ & \\
Urban & $3(12 \%)$ & $18(90 \%)$ & \\
Rural & $3(12 \%)$ & $26(86.7 \%)$ & 1 \\
Consanguinity & $2(10 \%)$ & & \\
Positive & $4(13.3 \%)$ & $24(88.9 \%)$ & \\
Negative & & $20(87 \%)$ & \\
Family history & $3(11.1 \%)$ & & 0.277 \\
Positive & $3(13 \%)$ & 0.000 & 0.000 \\
Negative & & & \\
Duration & $4.5 \pm(2.3)$ & & \\
Mean (SD) & $16.2 \pm(1.3)$ & & \\
HbAlc level & & & \\
Mean (SD) & & & \\
\hline
\end{tabular}

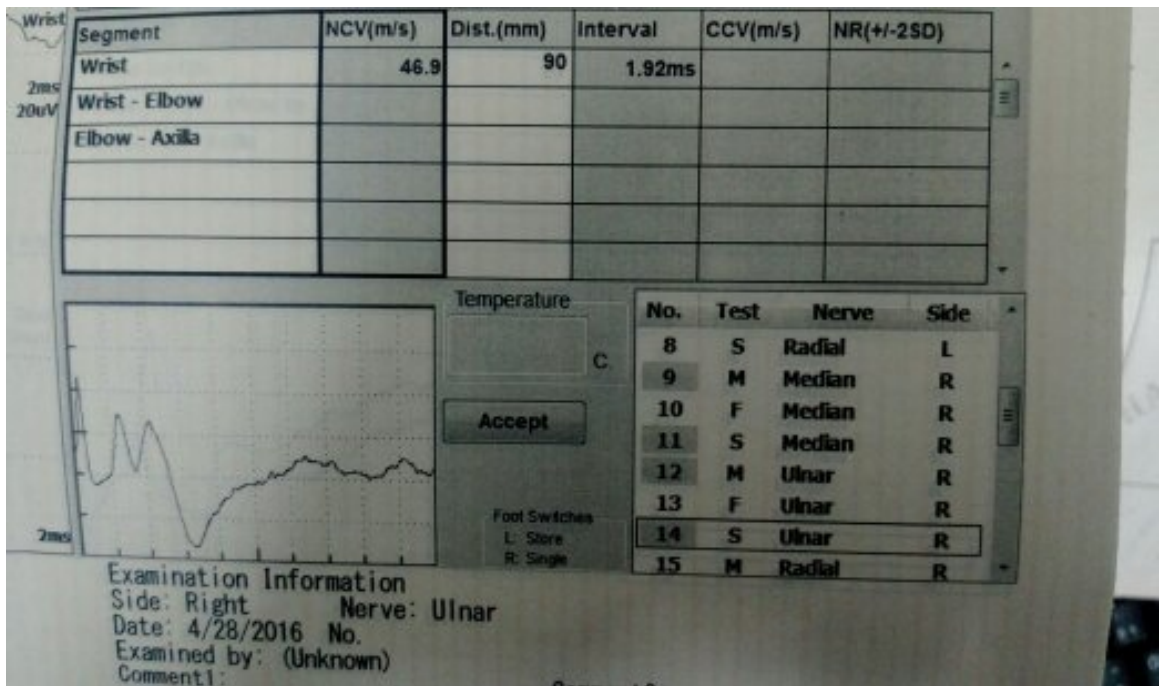

Image 1. Case no. (1). 


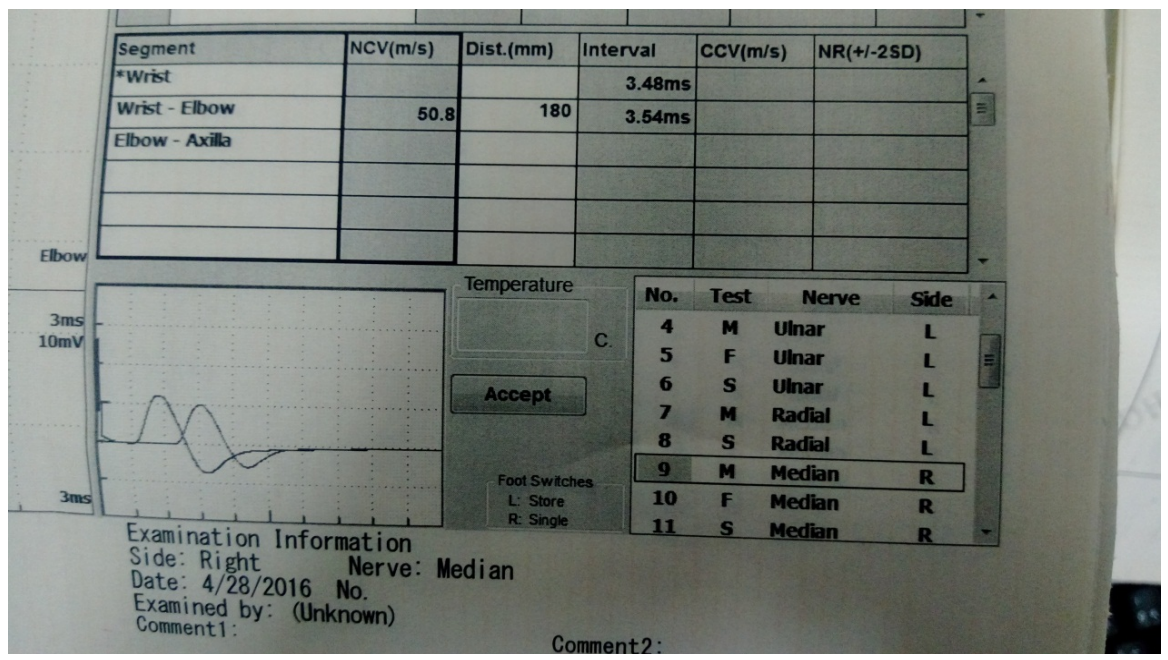

Image 2. Case no. (2).

Alc was 13 with normal fundus examination, diagnosed by NCS to have Rt. minimal sensory carpal tunnel syndrome (CTS) with impaired 2 sensory comparative studies on Rt. side.

Image 2: NCS show bilateral mild CTS more on Rt. Side of a male diabetic patient 16 years old diagnosed to have diabetes 3 years ago, was complaining of upper limb numbness and tingling and lower limb weakness, his serum creatinine was $0.7, \mathrm{HbAlc}$ was 12 with refractive errors and normal fund us examination, diagnosed by NCS to have bilateral mild CTS more on Rt. Side with comparative study done between median and ulnar nerve found that median nerve more affected than ulnar nerve $(>0.6)$.

\section{Discussion}

DPN is a chronic complication of the diabetes that is rarely reported in children, since it has a relatively longer latency period [13] [14]. Our main objective in this study was to determine the prevalence of DPN among diabetic children and to assess the percent of subclinical neuropathy in addition to identification of possible risk factors of DPN among these children.

Our findings showed that $12 \%$ of the studied diabetic children had DPN. These findings are in agreement with [15] and [16] who reported a prevalence rate of $11 \%$ and $13.75 \%$. However Effat et al., reported much higher prevalence (23\%) among the studied children having TIDM [17]. Moreover Hollner et al. reported that the incidence of DN was $15 \%$ in symptomatic DN cases and $38 \%$ in DN cases diagnosed by NCS [18]. Their conclusion was that in type 1DM that is seen in children and adolescents, DPN had a higher prevalence rate; however, the majority of cases remain subclinical. But in our study the majority of diagnosed cases had symptoms of DPN in addition to their diagnosis by Nerve Conduction Analysis.

DPN can be classified into two broad subclinical and clinical stages. Based on results of previous studies, the prevalence of diabetic neuropathy in developing 
countries ranged between $10 \%$ and 68\% [19] [20] and in Western countries, up to $60 \%$ of all patients with diabetes are affected by DPN [21]. However, the estimated rates of children are inaccurate, considering the prevalence of subclinical diabetic neuropathy observation in this age group.

Based on results of NCV and compared with clinical examination of the studied diabetic children, we found that $2 \%$ of diabetic children had subclinical neuropathy, they showed no symptoms or signs of neuropathy on clinical examination. On the other hands these cases of subclinical neuropathy constituted $16 \%$ of the total diagnosed cases of DPN. In agreement with our findings but with much more percentages of subclinical neuropathy were the findings of Toopchizadeh et al,, who reported that among the diagnosed cases of DPN (57.5\%) showed no symptoms of neuropathy [20]. Moreover, Nelson et al. [5] found subclinical neuropathy based on nerve conduction findings in $57 \%$ of Canadian children and adolescents with at least 5 years duration of T1DM. Baba and Ozaki found polyneuropathy using neurologic examinations in $43 \%$ of the studied diabetic children and using electro diagnostic studies in $63 \%$ of children and adolescents without clinical neuropathy [22]. These results indicate that, routine nerve conduction velocity $(\mathrm{NCV})$ assessment for $\mathrm{DN}$ is beneficial in those patient groups [18].

The lack of standardization of screening methods seems to be a general problem in diagnostic research on DPN There is considerable uncertainty about the prevalence of diabetic neuropathy (DN) due to a lack of large epidemiological studies and consensus on diagnostic criteria.

As regard factors associated with occurrence of DPN among the studied diabetic children, in agreement with [20], no significant relation was found for age gender, residence, consanguinity and family history.

Association between DPN and duration of diabetes had been assessed and reported by many previous studies especially in adolescents and adults, however in children this association remains a matter of controversy. On contrary to [5] [23], we found no significant association between duration of diabetes and development of DPN. Although these findings agree with [20] [24] [25].

The level of glycaemic control in the studied diabetic children was unsatisfactory (a mean of 8.9 among the normal group and 16.2 among the group having DN). As recommended by the International Society for Pediatric and Adolescent Diabetes (ISPAD) a level of $<7.5 \%$ (HbAlc) is crucial for a good metabolic control for all age-groups. As long-term good metabolic control reduces the total risk of complications in type $1 \mathrm{DM}$ [26]. In agreement with our finding several previous researches [23] [25] [27] demonstrated that poor glycaemic control contribute significantly in development of $\mathrm{DN}$ among diabetic children.

\section{Conclusion}

Based on the results of the current study, subclinical peripheral neuropathy is frequent complication in diabetic children that related to poor glycaemic control 
rather than duration of the disease. This indicates the need for annual screening of neuropathy in children with TIDM using Nerve Conduction Study due to poor cooperation of these children as regard neurologic examination. Moreover, strict glycaemic control is needed to prevent rapid development of peripheral neuropathy and other complication among these children.

\section{Limitation of the Study}

The major limitation of the study was the reduced no of children with TI DM. This can be explained by the fact that NCA is a painful investigation that needs cooperation of the child. And many children refused to complete the test.

\section{Conflicts of Interest}

The authors declare no conflicts of interest regarding the publication of this paper.

\section{References}

[1] Nathan, D.M., Davidson, M.B., DeFronzo, R.A., Heine, R., Henry, R.R., Pratley, R., et al. (2007) Impaired Fasting Glucose and Impaired Glucose Tolerance: Implications for Care. Diabetes Care, 30, 753-759. https://doi.org/10.2337/dc07-9920

[2] Diana, K. (2014) Trials in Prevention of Type 1 Diabetes: Current and Future. Canadian Journal of Diabetes, 38, 279-284. https://doi.org/10.1016/j.jcjd.2014.05.004

[3] International Diabetes Federation (2013) IDF Diabetes Atlas. 6th Edition, Brusseles, 279-284. http://www.idf.org/diabetesatla

[4] Al Bahnasy, R.E., Mahrous, O.A., El Shazli, H.M., Gabr, H.M., Ibrahem, R.A. and Soliman, S.S. (2017) Prevalence of Diabetes Mellitus and Impaired Glucose Tolerance among Adolescents in Menoufia Governorate, Egypt. Menoufia Medical Journal, 30, 69-75. https://doi.org/10.4103/1110-2098.211493

[5] Nelson, D., Mah, J.K., Adams, C., Hui, S., Crawford, S., Darwish, H., et al. (2006) Comparison of Conventional and Non-Invasive Techniques for the Early Identification of Diabetic Neuropathy in Children and Adolescents with Type 1 Diabetes. Pediatric Diabetes, 7, 305-310. https://doi.org/10.1111/j.1399-5448.2006.00208.x

[6] Abbott, C.A., Carrington, A.L., Ashe, H., Bath, S., Every, L.C., Griffiths, J., et al. (2002) The North-West Diabetes Foot Care Study: Incidence of, and Risk Factors for, New Diabetic Foot Ulceration in a Community-Based Patient Cohort. Diabetic Medicine, 19, 377-384. https://doi.org/10.1046/j.1464-5491.2002.00698.x

[7] Vinik, A. (2006) Neuropathies in Children and Adolescents with Diabetes: The Tip of the Iceberg. Pediatric Diabetes, 7, 301-304. https://doi.org/10.1111/j.1399-5448.2006.00213.x

[8] Mantovani, A.M., Fregonesi, C.E., Palma, M.R., Ribeiro, F.E., Fernandes, R.A. and Christofaro, D.G. (2017) Relationship between Amputation and Risk Factors in Individuals with Diabetes Mellitus: A Study with Brazilian Patients. Diabetes \& Metabolic Syndrome: Clinical Research and Reviews, 11, 47-50. https://doi.org/10.1016/j.dsx.2016.08.002

[9] Malgrange, D., Richard, J.L., Leymarie, F. and French Working Group on the Diabetic Foot (2003) Screening Diabetic Patients at Risk for Foot Ulceration. A Multi-Centre Hospital-Based Study in France. Diabetes \& Metabolism, 29, 261-268. 
https://doi.org/10.1016/S1262-3636(07)70035-6

[10] Aring, A.M., Jones, D.E. and Falko, J.M. (2005) Evaluation and Prevention of Diabetic Neuropathy. American Family Physician, 71, 2123-2128.

[11] Javadzadeh, A., Ghorbanihaghjo, A., Adl, F.H., Andalib, D., Khojasteh-Jafari, H. and Ghabili, K. (2013) Calcium Dobesilate Reduces Endothelin-1 and High-Sensitivity C-Reactive Protein Serum Levels in Patients with Diabetic Retinopathy. $\mathrm{Mo}$ lecular Vision, 19, 62-68.

[12] Samahy, M.H., Elbarbary, N.S. and Elmorsi, H.M. (2015) Current Status of Diabetes Management, Glycemic Control and Complications in Children and Adolescents with Diabetes in Egypt. Where Do We Stand Now? And Where Do We Go from Here? Diabetes Research and Clinical Practice, 107, 370-376. https://doi.org/10.1016/j.diabres.2015.01.004

[13] Neu, A., Beyer, P., Bürger-Büsing, J., et al. (2011) Diagnosis, Treatment and Follow-Up of Diabetes Mellitus in Childhood and Adolescence. Diabetologie und Stoffwechsel, 6, 159. (In German) https://doi.org/10.1055/s-0031-1283769

[14] Craig, M.E., Twigg, S.M., Donaghue, K.C., et al. (2011) National Evidence-Based Clinical Care Guidelines for Type 1 Diabetes in Children, Adolescents and Adults. Australian Government Department of Health and Ageing, Canberra.

[15] Mosera, J.T., Langdon, D.R., et al. (2013) The Evaluation of Peripheral Neuropathy in Youth with Type 1 Diabetes. Diabetes Research and Clinical Practice, 100, e3-e6. https://doi.org/10.1016/j.diabres.2013.01.015

[16] Karamifar, H., Mooadab, M., Karamizadeh, Z. and Amirhakimi, G. (2007) Evaluation of Peripheral Neuropathy in Patients with Type 1 Diabetes Mellitus by Bedside Scoring Procedure. Iranian Journal of Pediatrics, 17, 54-60.

[17] Khorasani, E. (2018) Prevalence of Peripheral Neuropathy and Its Related Factors in Diabetic Children, Neishabour City, Iran. International Journal of Pediatrics, 6, Serial No. 60.

[18] Holiner, I., Haslinger, V., Lütschg, J., Müller, G., Barbarini, D.S., Fussenegger, J., Zanier, U., Saely, C.H., Drexel, H. and Simma, B. (2013) Validity of the Neurological Examination in Diagnosing Diabetic Peripheral Neuropathy. Pediatric Neurology, 49, 171-177. https://doi.org/10.1016/j.pediatrneurol.2013.03.014

[19] Trotta, D., Verrotti, A., Salladini, C. and Chiarelli, F. (2004) Diabetic Neuropathy in Children and Adolescents. Pediatric Diabetes, 5, 44-57. https://doi.org/10.1111/j.1399-543X.2004.00041.x

[20] Toopchizadeh, V., Shiva, S., Khiabani, N.-Y. and Ghergherechi, R. (2016) Electrophysiologic Pattern and Prevalence of Subclinical Peripheral Neuropathy in Children and Adolescents with Type I Diabetes Mellitus in Iran. Saudi Medical Journal, 37, 299-303. https://doi.org/10.15537/smj.2016.3.13625

[21] Sajic, S. and Petrovic, R. (2005) Subclinical Peripheral Neuropathy in Type 1 Diabetic Adolescents and Its Relationship with Metabolic Control. Srpski Arhiv za Celokupno Lekarstvo, 133, 358-362. https://doi.org/10.2298/SARH0508358S

[22] Baba, M. and Ozaki, I. (2001) Electrophysiological Changes in Diabetic Neuropathy: From Subclinical Alterations to Disabling Abnormalities. Archives of Physiology and Biochemistry, 109, 234-240. https://doi.org/10.1076/apab.109.3.234.11595

[23] Booya, F., Bandarian, F., Larijani, B., Pajouhi, M., Nooraei, M. and Lotfi, J. (2005) Potential Risk Factors for Diabetic Neuropathy: A Case Control Study. BMC Neurology, 5, 24. https://doi.org/10.1186/1471-2377-5-24

[24] Karsidag, S., Morali, S., Sargin, M., Salman, S., Karsidag, K. and Us, O. (2005) The 
Electrophysiological Findings of Subclinical Neuropathy in Patients with Recently Diagnosed Type 1 Diabetes Mellitus. Diabetes Research and Clinical Practice, 67, 211-219. https://doi.org/10.1016/j.diabres.2004.07.017

[25] Ghaemi, N., Hasanabadi, H., Ashrafzadeh, F., Sarvari, S., Rahimi, H. and Hashemian, S. (2018) Peripheral Neuropathy in Children and Adolescents with Insulin-Dependent Diabetes Mellitus. Iranian Journal of Child Neurology, 12, 83-90.

[26] Rewers, M., Pihoker, C., Donaghue, K., Hanas, R., Swift, P. and Klingensmith, G.J. (2009) ISPAD Clinical Practice Consensus Guidelines 2009 Compendium: Assessment and Monitoring of Glycemic Control in Children and Adolescents with Diabetes. Pediatric Diabetes, 10, 71-81.

https://doi.org/10.1111/j.1399-5448.2009.00582.x

[27] Ziegler, D., Behler, M., Schroers-Teuber, M. and Roden, M. (2015) Near-Normoglycaemia and Development of Neuropathy: A 24-Year Prospective Study from Diagnosis of Type 1 Diabetes. BMJ Open, 5, 1-9.

https://doi.org/10.1136/bmjopen-2014-006559 


\section{Questionnaire}

\section{Part I}
1) Age:
2) Gender: a) boy
b) girl
3) Parental consanguinity:
a) Yes
b) No
4) Residence:
a) urban
b) rural

5) Age of onset of Diabetes:

6) duration:

7) Symptoms of peripheral neuropathy: numbness, tingling, loss of sensation, ulceration
8) Family history of diabetes mellitus:
a) Yes
b) No
9) Family history of diabetic neuropathy:
a) Yes
b) No

\section{Part II}

Examination of neurological system:

10) Vibration perception:

11) temperature sensation:

12) pinprick sensation:

13) MMT and ankle reflex:

14) A tuning fork $(128 \mathrm{~Hz})$ examination:

15) Semmes-Weinstein monofilament test:

16) cold and warm water test:

\section{Part III}

NCV results of

17) Median nerve:

18) radial nerve:

19) ulnar nerves:

20) Sural nerve:

21) superficial peroneal:

21) results of Hemoglobin A1C: 